\title{
BMJ Open Comparing the efficacy and safety of faecal microbiota transplantation with bezlotoxumab in reducing the risk of recurrent Clostridium difficile infections: a systematic review and Bayesian network meta-analysis of randomised controlled trials
}

\author{
Abdullah A Alhifany, ${ }^{1}$ Abdulaali R Almutairi, ${ }^{2}$ Thamer A Almangour, ${ }^{3}$ \\ Alaa N Shahbar, ${ }^{1}$ Ivo Abraham, ${ }^{2}$ Mohammed Alessa, ${ }^{4}$ Faris S Alnezary, ${ }^{5}$ \\ Ejaz Cheema (1) ${ }^{6}$
}

To cite: Alhifany AA, Almutairi AR, Almangour TA, et al. Comparing the efficacy and safety of faecal microbiota transplantation with bezlotoxumab in reducing the risk of recurrent Clostridium difficile infections: a systematic review and Bayesian network meta-analysis of randomised controlled trials. BMJ Open 2019;9:e031145. doi:10.1136/ bmjopen-2019-031145

- Prepublication history and additional material for this paper are available online. To view these files, please visit the journal online (http://dx.doi org/10.1136/bmjopen-2019031145).

Received 18 April 2019 Revised 16 September 2019 Accepted 11 October 2019

Check for updates

(C) Author(s) (or their employer(s)) 2019. Re-use permitted under CC BY-NC. No commercial re-use. See rights and permissions. Published by BMJ.

For numbered affiliations see end of article.

Correspondence to

Dr Ejaz Cheema;

E.Cheema@bham.ac.uk

\section{ABSTRACT}

Objectives The risk of recurrent Clostridium difficile infections (RCDIs) is high when treated with standard antibiotics therapy (SAT) alone. It is suggested that the addition of faecal microbiota transplantation (FMT) or bezlotoxumab after SAT reduces the risk of RCDI. In the absence of head-to-head randomised controlled trials (RCTs), this review attempts to compare the efficacy and safety of bezlotoxumab with FMT in reducing the risk of RCDI in hospitalised patients.

Design A systematic review and Bayesian network metaanalysis.

Data source A comprehensive search from inception to 30 February 2019 was conducted in four databases (Medline/PubMed, Embase, Scopus, ClinicalTrials.gov). Eligibility criteria RCTs reporting the resolution of diarrhoea associated with RCDI without relapse for at least 60 days after the end of treatments as the primary outcome.

Data extraction and synthesis We extracted author, year of publication, study design and binomial data that represented the resolution of diarrhoea or adverse events of monoclonal antibodies and FMT infusion. Randomeffects models were used for resolution rate of RCDI and adverse events. The Cochrane Risk of Bias tool was used to assess the quality of included RCTs.

Results Out of 1003 articles identified, seven RCTs involving 3043 patients contributed to the review. No difference was reported between single or multiple infusions of FMT and bezlotoxumab in resolving RCDI, (OR 1.53, 95\% credible interval (Crl) 0.39 to 5.16 ) and (OR 2.86, 95\% Crl 1.29 to 6.57), respectively. Patients treated with SAT alone or bezlotoxumab with SAT showed significantly lower rates of diarrhoea than FMT (OR 0, 95\% Crl 0 to 0.09 ) and (OR 0, 95\% Crl 0 to 0.19 ), respectively. There was no difference in terms of other adverse events. Conclusions This is the first network meta-analysis that has compared the recently Food and Drug Administration-
Strengths and limitations of this study

Safety outcomes were limited due to the early termination of most of the included randomised controlled trials (RCTs) and the inconsistent reporting of the adverse events.

- The quality of the included RCTs varied with more than half of the studies not reporting blinding of the participants.

- The study employed a comprehensive literature search of four databases.

- It used Bayesian estimation methods in the indirect comparisons of monoclonal antibodies and faecal microbiota transplantation to address the absence of head-to-head clinical trial evidence.

approved monoclonal antibody bezlotoxumab with FMT for resolving RCDI. The quality of the included RCTs was variable. The findings of this study suggested no difference between single or multiple infusions of FMT and bezlotoxumab. However, FMT was associated with a higher rate of non-serious diarrhoea as opposed to SAT used alone or in combination with bezlotoxumab.

\section{BACKGROUND}

Clostridium difficile is considered to be the most common source of infectious diarrhoea in hospitalised patients. ${ }^{1}$ C. difficile-led infections (CDIs) are associated with high mortality particularly in the developed countries, including USA, Canada and Europe. $^{2-4}$ Around $30 \%$ of the C. difficileinfected patients treated with standard antibiotics therapy (SAT), such as vancomycin, metronidazole or fidaxomicin, are reported 
to develop recurrent CDIs (RCDIs) that increase up to $60 \%$ with subsequent recurrences. ${ }^{5}$ This cyclic pattern of recurring CDI-inducing diarrhoea is triggered by the use of antibiotics and exotoxins produced by $C$. difficile that contributes to the weakening of the intrinsic faecal microbiota which serves as a natural host defense mechanism against C. difficile spores-led colonisation. ${ }^{5-7}$ The sporeforming ability of C. difficile is the main reason behind its nosocomial and community transmission.

Faecal microbiota transplantation (FMT) has been considered a novel intervention to replenish the intrinsic faecal microbiota barrier mechanism that protects against C. difficile-associated colonisation. ${ }^{8}$ Evidences from the meta-analyses of randomised controlled trials (RCTs) as well as observational studies have highlighted the benefits of FMT in resolving CDI over SAT alone ${ }^{9-12}$ Furthermore, the current clinical practice guidelines by the Infectious Diseases Society of America (IDSA) and the Society for Healthcare Epidemiology of America recommend the use of FMT for the second or subsequent recurrences of CDI. ${ }^{13}$ However, the lack of a standardised product, dosage form and method of administration are some of the limitations of FMT. ${ }^{14}$

An alternative approach to FMT is to attenuate the effects of the exotoxins produced by $C$. difficile. Bezlotoxumab, a novel monoclonal antibody (mAB) that has been approved recently by the Food and Drug Administration (FDA) in the USA, has been reported to reduce RCDI by attenuating the effect of exotoxin B when used in conjunction with SAT. ${ }^{15}{ }^{16}$ However, there are no headto-head clinical trials that have compared the efficacy and safety of FMT with bezlotoxumab in reducing the risk of RCDI. In the absence of any head-to-head trials, this systematic review and Bayesian network meta-analysis of RCTs aims to compare the efficacy and safety of bezlotoxumab with FMT in reducing the risk of RCDI. The review would attempt to determine if FMT when compared with bezlotoxumab has better efficacy and safety in resolving the diarrhoea associated with CDI in hospitalised patients without relapse or not.

\section{METHOD}

The systematic review and network meta-analysis were conducted according to the Preferred Reporting Items for Systematic Reviews and Meta-Analyses extension statement for network meta-analyses. ${ }^{17}$

\section{Patient and public involvement}

Patients and public were not involved in the design, conducting and reporting of research.

\section{Search strategy}

A comprehensive search from inception to 30 February 2019 was conducted in four databases (Medline/PubMed, Embase, Scopus, ClinicalTrials.gov). Searches were conducted using Patients, Intervention, Comparator, Outcome and Study design strategy for clinical evidence
Table 1 PICOS strategy for clinical evidence of FMT and $\mathrm{mAB}$ in $\mathrm{CDI}$

\begin{tabular}{|c|c|}
\hline PICOS & Clinical review \\
\hline Population & Adultswith primary or RCDI. \\
\hline Intervention & $\begin{array}{l}\text { Studies that reported the efficacy and safety } \\
\text { of FMT and/or mABs at any dosage form and } \\
\text { via any route of administration in resolving } \\
\text { RCDI. }\end{array}$ \\
\hline Comparator & $\begin{array}{l}\text { Standard antibiotics therapy, such as } \\
\text { vancomycin, metronidazole, orfidaxomicin, } \\
\text { at any dosage form and via any route of } \\
\text { administration. }\end{array}$ \\
\hline Outcome & $\begin{array}{l}\text { The resolution of diarrhoea associated to CD } \\
\text { without relapse for, at least, } 60 \text { days after the } \\
\text { end of treatments. } \\
\text { Adverse events. }\end{array}$ \\
\hline Study design & $\begin{array}{l}\text { Published or unpublished randomised } \\
\text { controlled trials of any size and duration. }\end{array}$ \\
\hline
\end{tabular}

CDI, Clostridium difficile infection; FMT, faecal microbiota

transplantation; $\mathrm{mAB}$, monoclonal antibody.

of FMT and $\mathrm{mAB}$ in CDI (table 1) (see online supplementary file for the complete search strategy). Furthermore, manual searches were conducted to identify any additional studies by checking the reference lists of articles retrieved.

\section{Outcome measure}

The primary outcome of interest was the resolution of diarrhoea associated with CDI without relapse for at least 60 days after the end of treatments. Furthermore, the adverse events of interest included diarrhoea, abdominal pain, leucocytosis, fatigue, nausea, fever, atrial fibrillation, dehydration, sepsis, tachycardia and infusion-specific reactions.

\section{Inclusion and exclusion criteria}

Both published as well as unpublished RCTs that assessed the efficacy and safety of FMT and bezlotoxumab in resolving CDI after a short course of SAT such as vancomycin, metronidazole or fidaxomicin were eligible for inclusion. Studies were eligible for inclusion if they had included patients 18 years or older diagnosed with RCDI and reported the resolution rate of CDI as the efficacy outcome.

\section{Data extraction, risk of bias and quality assessment}

Two reviewers (EC and ANS) independently reviewed the titles and abstracts. Studies meeting the inclusion criteria were retrieved as full text to further assess their eligibility for inclusion. Reviewer, AAA, independently extracted data from included studies using a data extraction sheet (see table 2 for characteristics of included studies). Reviewer, ANS, checked all data extracted in the sheets. The data extracted included; author, year of publication, study design and clinical data reporting resolution outcomes of mABs and FMT infusion. The Cochrane 


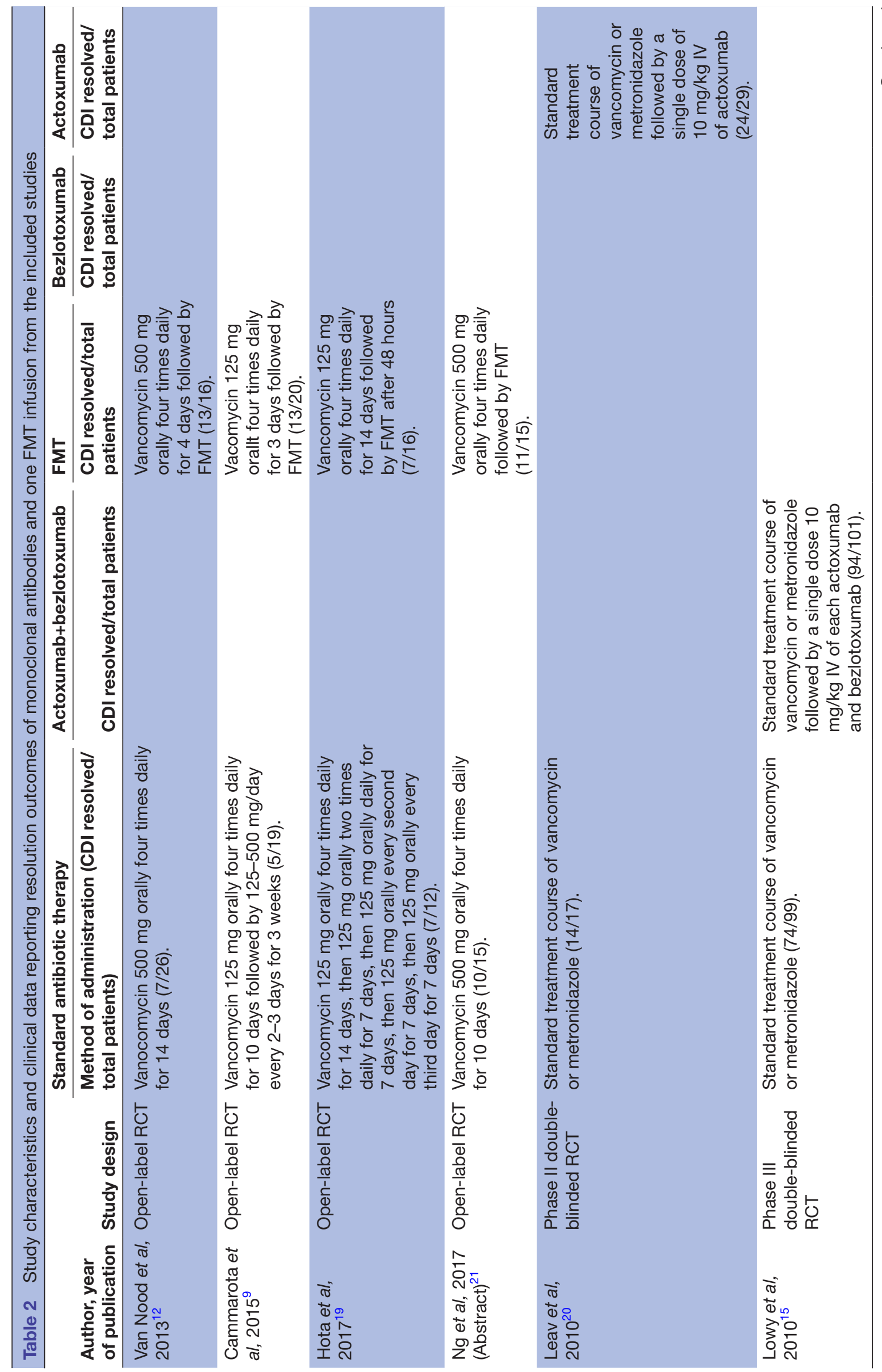




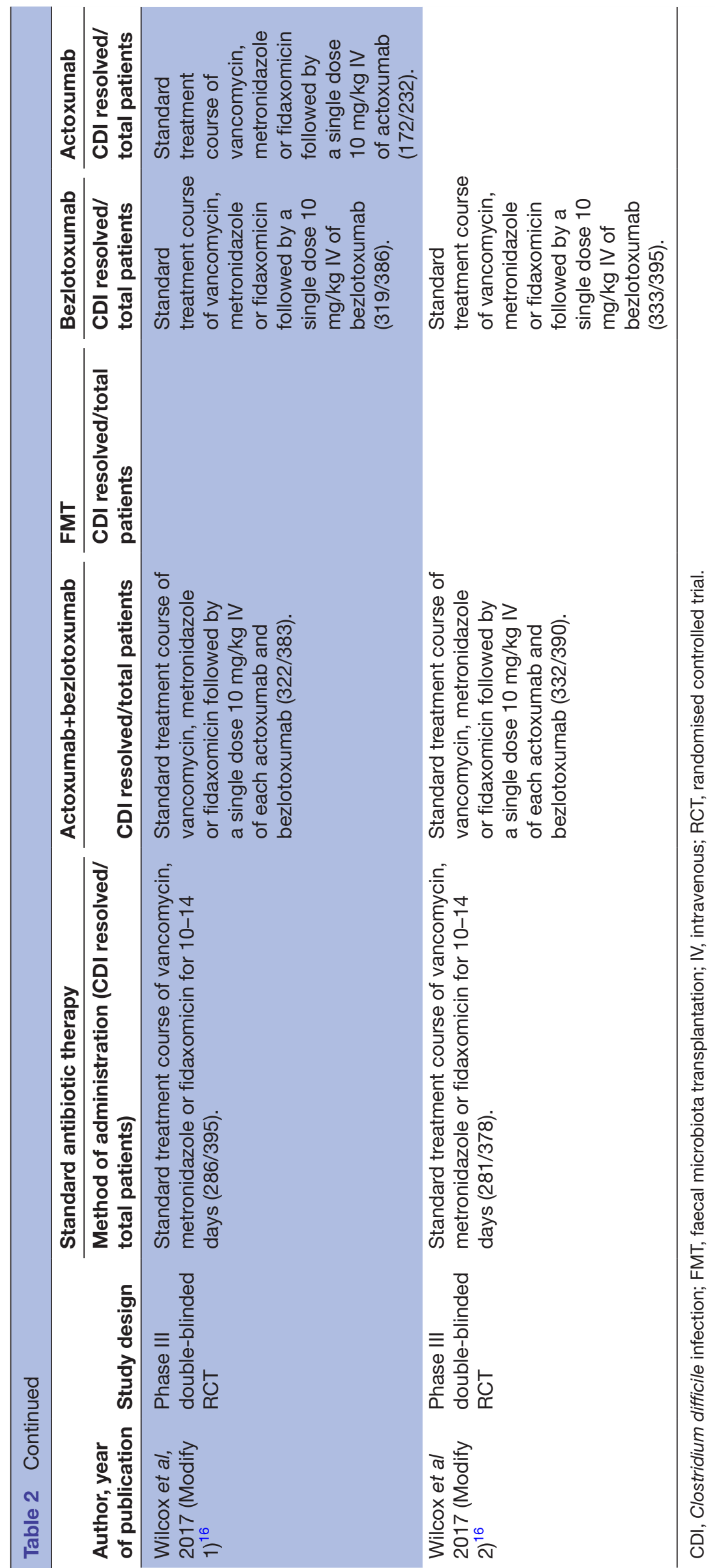




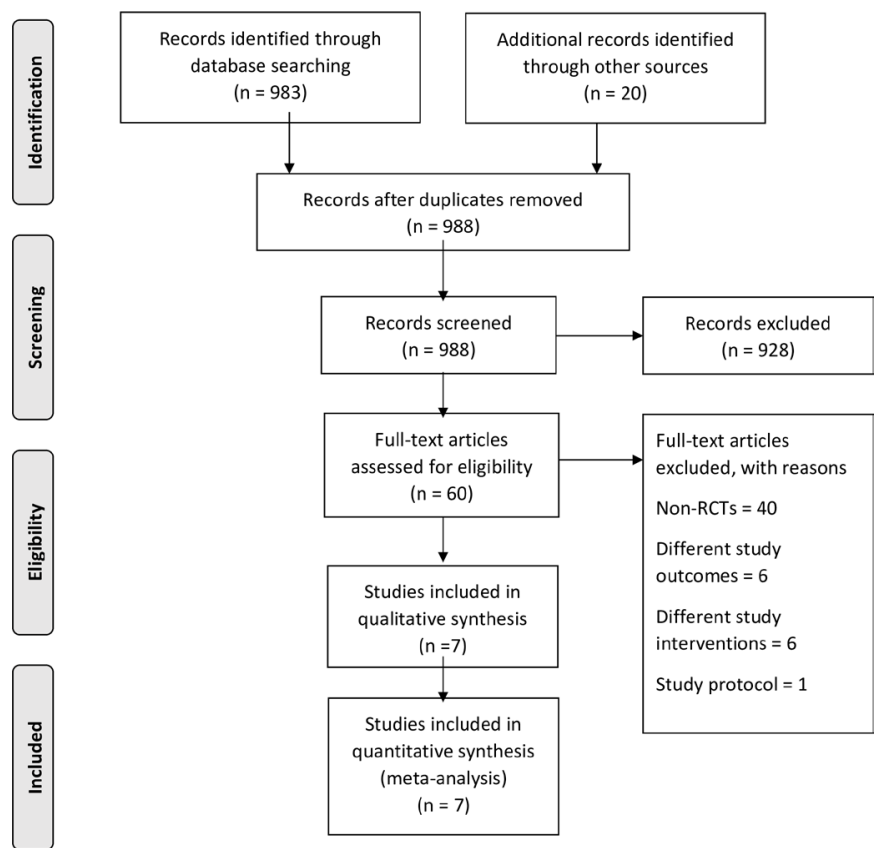

Figure 1 Study selection process using Preferred Reporting Items for Systematic Reviews and Meta-analyses. RCT, randomised controlled trial.

Risk of Bias tool was used to assess the quality of included RCTs including randomisation, allocation concealment, blinding of participants, reporting of incomplete outcome data, selective reporting and any other bias. ${ }^{18}$ Other sources of bias explored included cross-contamination between-study groups, recruitment of participants from a selected population and non-compliance with the study protocol. For each included study, a risk of bias graphs and risk of bias summary were generated.

\section{Statistical analysis}

A Bayesian network meta-analysis was conducted using WinBUGS V.1.4.3 (MRC Biostatistics Unit, Cambridge, UK). Binomial data that represented the resolution of diarrhoea or adverse events were extracted and analysed. The binary outcomes were expressed as OR and $95 \%$ credible interval $(95 \% \mathrm{CrI})$ for resolution rate of RCDI and OR with 95\% CrI for adverse events. ORs for treatment comparisons were estimated based on 20000 iterations, following the discarding of the first 10000 iterations in the model. Random-effects model was used for the resolution rate of RCDI and adverse events due to the assumed variability between the included studies. A binomial likelihood with a logit link was used in the model. Furthermore, non-informative priors were used for all parameters.Ranking probabilities of treatments were calculated using the surface under the cumulative ranking curve (SUCRA) method. Additionally, a sensitivity analysis was conducted to exclude the studies and/or patients who received non-FDA-approved mAB. Furthermore, a pairwise meta-analysis was conducted to check for heterogeneity.

\section{RESULTS}

The initial search identified 1003 studies (see figure 1). Fifteen duplicates along with an additional 631 studies were removed at title level due to not meeting the inclusion criteria. The abstracts of remaining 357 studies were reviewed, of which a further 297 were excluded. Full texts of the remaining 60 studies were reviewed. Of these, further 53 studies were excluded based on the inclusion and exclusion criteria. The remaining 7 RCTs with 3043 patients contributed to the review and network meta-analysis.

\section{Characteristics of included studies}

The seven included studies (see table 2 for characteristics of included studies) were published between 2010 and 2017 and involved 3043 patients. $^{9} 12151619-21$ Four openlabelled RCTs involving 139 patients reported comparisons of FMT versus vancomycin alone in patients with an initial episode of CDI or with RCDI and followed for at least 70 days following the end of the treatments. ${ }^{9} 121921$ Patients assigned to the FMT arm received an initial course of vancomycin ranging from 3 to 14 days to assure that patients were covered with an antibiotic therapy at the time of donor screening.

All studies involving FMT used fresh faeces from related donors. The time for infusing the fresh FMT from the time of defaecation varied across studies from 3.1 to 48 hours. Three FMT studies were terminated early following an interim analysis; two because of the observed superiority of the $\mathrm{FMT}^{912}$ and one because of inferiority. ${ }^{19}$

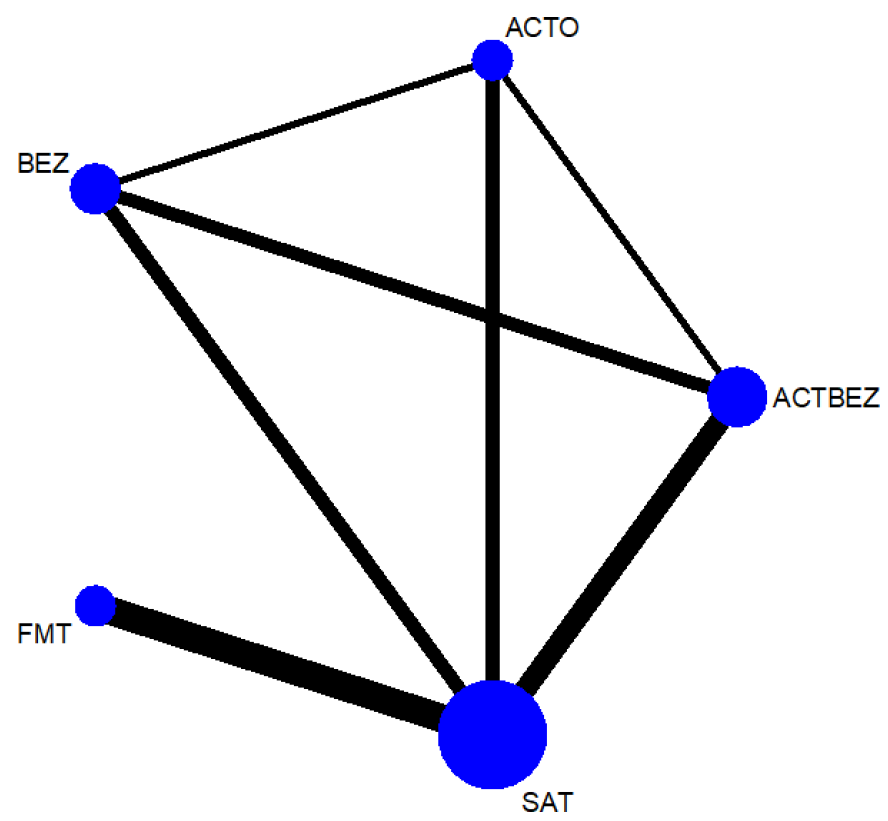

Figure 2 Network plot of included studies. Each circled node represents an intervention, the extent of the circle indicates the number of the included participants, the lines and their thickness represent direct comparisons and the number of studies included in each comparison, respectively. ACTBEZ, actoxumab plus bezlotoxumab; ACTO, actoxumab; BEZ, bezlotoxumab; FMT, faecal microbiota transplantation; SAT, standard antibiotics therapy. 
A fourth study was underpowered and was considered a pilot study. ${ }^{21}$ In two studies, ${ }^{912}$ FMT was reinfused in some patients who experienced a recurrence after the first infusion. In the remaining two studies, FMT was used as a single infusion. ${ }^{19} 21$

Three double-blinded, placebo-controlled RCTs including two multicentre phase II studies and one multinational, multicentre phase III study investigated the efficacy of mABs. ${ }^{151620}$ The two phase two studies investigated the safety and efficacy of newly developed mABs against $C$. difficile exotoxins $\mathrm{A}$ and $\mathrm{B}$ that corroborated prior evidence of the role of these exotoxins in the virulence of $C$. difficile. ${ }^{6}$ The third RCT confirmed that antagonising toxin $B$ is the main determinant in suppressing the virulence of $C$. difficile, however, it could not rule out the role of toxin $\mathrm{A}^{7}$ Three regimens of $\mathrm{mABs}$ were tested in these RCTs: antitoxin A (actoxumab), antitoxin B (bezlotoxumab) and a combination of both, all of which were infused as a single dose of $10 \mathrm{mg} / \mathrm{kg}$ either during or right away after a course of SAT. It is important to highlight that only bezlotoxumab was approved by the FDA for this indication (see figure 2 for network plot of included studies).

\section{Study quality}

The quality of the studies was variable (see figure 3A,B). Only three of the seven studies used blinding of participants. ${ }^{151620}$

\section{Comparative efficacy of FMT and $\mathrm{mABs}$ in reducing RCDI}

The initial analysis comparing the resolution of CDI after receiving one FMT infusion or any $\mathrm{mAB}$ regimen found

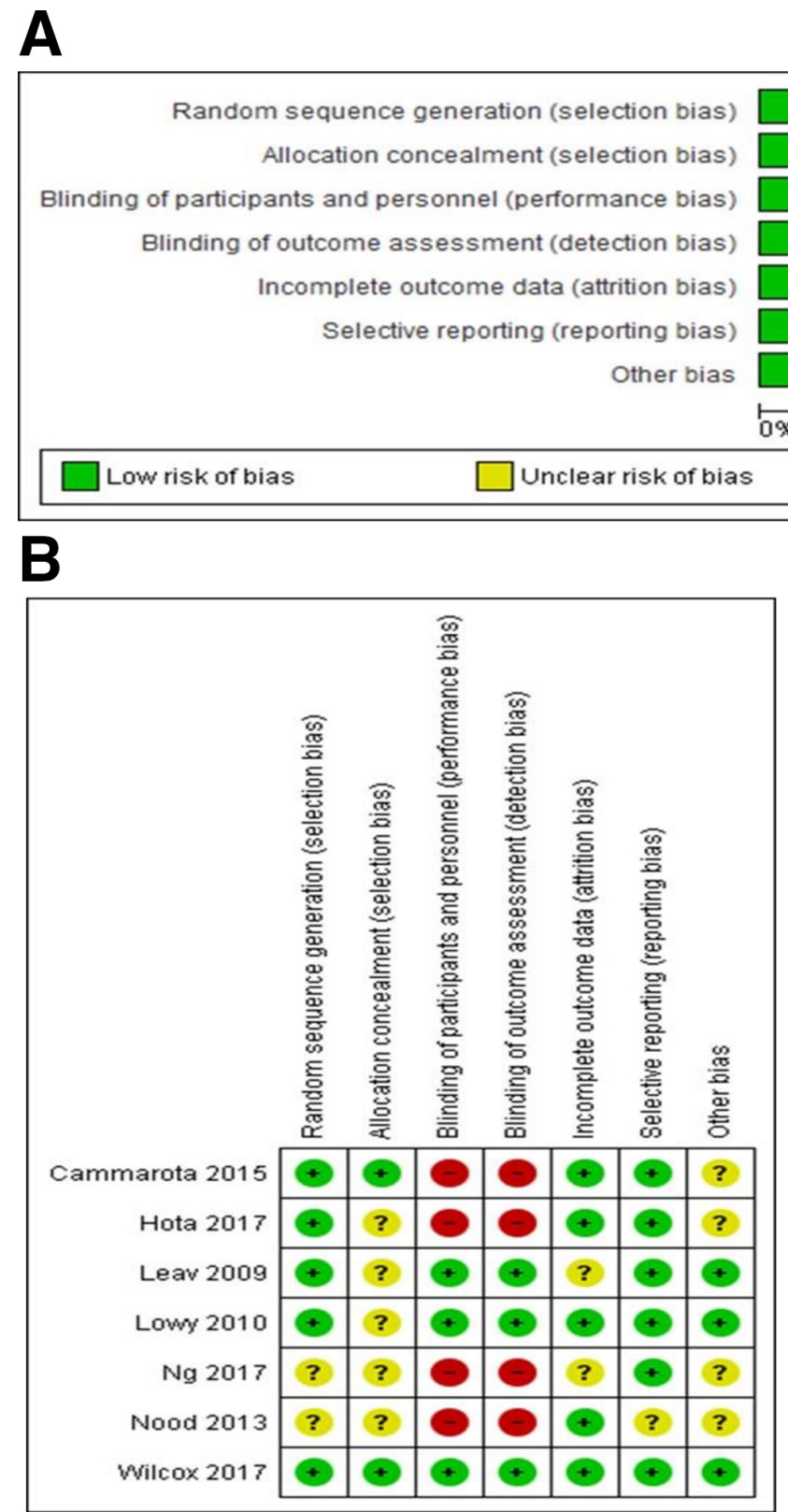

Figure 3 (A) Risk of bias graph: review authors' judgements about each risk of bias item presented as percentages across all included studies. (B) Risk of bias summary: review authors' judgements about each risk of bias item for each included study. 


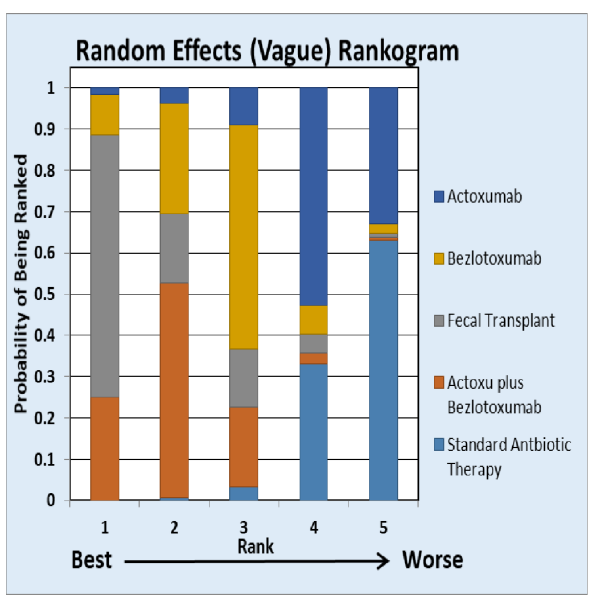

\begin{tabular}{|l|l|l|}
\hline Rank & Treatment & SUCRA \\
\hline 1 & Fecal Microbiota Transplantation & 0.6357 \\
\hline 2 & Actoxumab plus Bezlotoxumab & 0.5215 \\
\hline 3 & Bezlotoxumab & 0.5421 \\
\hline 4 & Actoxumab & \\
\hline 5 & Standard Antibiotics Therapy & 0.528 \\
& & 0.631 \\
\hline
\end{tabular}

Figure 4 Rankograms show the ranking of interventions from the best to the worst based on the corresponding surface under the cumulative ranking curve (SUCRA) probability in the random-effect models.

no statistical difference between FMT and bezlotoxumab (OR 1.53, 95\% CrI 0.39 to 5.16). Yet, FMT showed the best SUCRA probability (63.6\%) (see figure 4 ). In addition, FMT showed better resolution of CDI than SAT (OR 2.98, 95\% CrI 1.13 to 7.53). Whereas, bezlotoxumab showed no statistical difference in resolution of CDI than SAT (OR 1.93, 95\% CrI 0.84 to 4.91 ) (see table 3).

A secondary comparative analysis that included the resolution outcomes reported for patients who received two or more FMT infusions or any $\mathrm{mAB}$ regimen was conducted. FMT did not show statistical difference in resolution of CDI than bezlotoxumab (OR 2.58, 95\% CrI 0.30 to 23.53). In addition, bezlotoxumab showed no statistical difference in resolution of CDI than SAT (OR 2.01, 95\% CrI 0.40 to 10.51) However, FMT showed better resolution of CDI than SAT (OR 5.22, 95\% CrI 1.26 to 23.25) (see table 3).

The analyses of the safety data for FMT, bezlotoxumab and SAT revealed a significantly lower rate of non-serious diarrhoea in patients receiving bezlotoxumab (OR 0 , 95\% CrI 0 to 0.19 ) and SAT (OR 0, 95\% CrI 0 to 0.09 ), compared with patients treated with FMT. There were no differences on other adverse events.

\section{Sensitivity analysis}

A sensitivity analysis was conducted by excluding the resolution outcomes of patients who received non-FDAapproved mABs. There was no difference between single or multiple FMT infusion and bezlotoxumab (OR 1.61, $95 \%$ CrI 0.19 to 12.69 ), (OR 2.90, 95\% CrI 0.20 to 45.35 ), respectively. However, FMT showed the best SUCRA probability $(79.7 \%)$. The pairwise meta-analysis suggested that heterogeneity $\mathrm{I}^{2}$ for SAT versus FMT and for SAT versus mABs was high which indicated high variability between the studies.

\section{DISCUSSION}

To the best of authors' knowledge, this is the first network meta-analysis that has compared the recently FDAapproved monoclonal antibody bezlotoxumab with FMT for resolving RCDI. The findings of this study suggested that single or multiple infusions of FMT showed no difference in efficacy than a single infusion of bezlotoxumab. However, FMT was associated with a higher rate of nonserious diarrhoea.

\begin{tabular}{|c|c|c|c|c|}
\hline FMT & $\begin{array}{l}1.29 \\
(0.34-3.93)\end{array}$ & $\begin{array}{l}1.53 \\
(0.39-5.16)\end{array}$ & $\begin{array}{l}2.61 \\
(0.64-9.74)\end{array}$ & $\begin{array}{l}2.98 \\
(1.13-7.53)\end{array}$ \\
\hline $\begin{array}{l}2.10 \\
(0.28-15.99)\end{array}$ & $\begin{array}{l}\text { Actoxumab- } \\
\text { bezlotoxumab }\end{array}$ & $\begin{array}{l}1.17 \\
(0.50-3.12)\end{array}$ & $\begin{array}{l}2.01 \\
(0.74-6.42)\end{array}$ & $\begin{array}{l}2.28 \\
(1.15-5.52)\end{array}$ \\
\hline $\begin{array}{l}2.58 \\
(0.30-23.53)\end{array}$ & $\begin{array}{l}1.22 \\
(0.24-6.66)\end{array}$ & Bezlotoxumab & $\begin{array}{l}1.71 \\
(0.57-5.49)\end{array}$ & $\begin{array}{l}1.93 \\
(0.84-4.91)\end{array}$ \\
\hline $\begin{array}{l}4.55 \\
(0.49-45.11)\end{array}$ & $\begin{array}{l}2.14 \\
(0.33-15.50)\end{array}$ & $\begin{array}{l}1.75 \\
(0.24-13.43)\end{array}$ & Actoxumab & $\begin{array}{l}1.14 \\
(0.42-3.10)\end{array}$ \\
\hline $\begin{array}{l}5.22 \\
(1.26-23.25)\end{array}$ & $\begin{array}{l}2.46 \\
(0.62-10.68)\end{array}$ & $\begin{array}{l}2.01 \\
(0.40-10.51)\end{array}$ & $\begin{array}{l}1.16 \\
(0.20-6.39)\end{array}$ & Standard antibiotic therapy \\
\hline
\end{tabular}


Despite the inconsistency in the results of the four RCTs that studied FMT versus SAT in resolving RCDI, ${ }^{9} 121921$ the network analysis showed the superiority of FMT over SAT alone. This is consistent with the findings of previous meta-analyses of RCTs and observational studies. ${ }^{10} 11$ The inconsistency in the results of the four included studies may be attributed to their small sample sizes, lack of blinding and variability between them on the basis of the process of collecting donor faeces, preparation of FMT, lag time between faeces collection and infusion, method of administration and SAT regimen. Furthermore, as evident from the findings of a previous RCT, patients who received FMT monotherapy for an initial episode of CDI without receiving prior antibiotics, retained more bacteroidetes in their gut than patients treated with antibiotics. ${ }^{22}$ These findings confirm the effect of antibiotics in attenuating intrinsic microbiota. ${ }^{5}$ It may also explain the inferiority of FMT over SAT in the study when FMT was preceded by 14 days of antibiotics. ${ }^{19}$ On the contrary, administration of FMT earlier (after the second recurrence of CDI) as opposed to a late administration (after the third or subsequent recurrences) led to shorter length of hospital stay and fewer visits to the emergency department. ${ }^{23}$ Thus, the differences in the results of the individual studies included in the current network metaanalysis could have been due to the variability in starting FMT for initial CDI or RCDI and the inconsistency in the number of previous recurrences among included patients.

Bezlotoxumab showed a favourable efficacy and safety profile in preventing RCDI in two robust prospective, double-blinded, placebo-controlled RCTs. ${ }^{16}{ }^{24}$ Furthermore, the effect was sustained throughout the 3-month follow-up. ${ }^{16}$ Bezlotoxumab has a novel mechanism of action that reduces the possibility of RCDI, yet its high cost may limit its utilisation. ${ }^{16}{ }^{24}$ Furthermore, even though the network meta-analysis did not report any difference in the resolution rate between bezlotoxumab and FMT, the SUCRA probability score favoured FMT in the rankogram.

This review had some limitaions. The quality of the included RCTs was variable with more than half of the studies not reporting blinding of the participants. Furthermore, the RCTs studying FMT differed in design, donor selection, FMT preparation, follow-up time, lag time between faeces collection and infusion and lag time between antibiotics discontinuation and FMT infusion; while $\mathrm{mABs}$ were infused either during or right away after the discontinuation of antibiotics. None of the included RCTs reported the number of previous recurrences. Furthermore, safety outcomes were limited due to the early termination of most of the included RCTs and the inconsistent reporting of the adverse events. Nevertheless, the review employed a rigorous and comprehensive search strategy to identify relevant studies. Furthermore, it used the Bayesian estimation methods in the indirect comparisons of mABs and FMT to address the absence of head-to-head clinical trial evidence. By doing so, this review addressed a significant issue identified in the 2017 IDSA $^{13}$ guidelines by filling the gap in information concerning the best method in preventing RCDI and the role of FMT and mABs therapies. However, further studies are required to assess the efficacy, safety, cost and clinical implications of multiple infusions of bezlotoxumab.

\section{CONCLUSION}

Single of multiple infusions of FMT showed no difference in efficacy than single infusion of bezlotoxumab in resolving RCDI but with a higher rate of non-serious diarrhoea. Further studies are needed to investigate the efficacy and safety of using FMT as monotherapy for CDI, the possible attenuating effect of short-course antibiotics given before FMT and the clinical implications of multiple infusions of bezlotoxumab.

\section{Author affiliations}

${ }^{1}$ Pharmacy, Umm Al-Qura University, Makkah, Saudi Arabia

${ }^{2}$ Pharmacy, University of Arizona, Tucson, Arizona, USA

${ }^{3}$ Department of Clinical Pharmacy, College of Pharmacy, King Saud University, Riyadh, Saudi Arabia

${ }^{4}$ College of Pharmcy, King Saud bin Abdulaziz University for Health Sciences,

Riyadh, Saudi Arabia

${ }^{5}$ Pharmacy, University of Houston, Houston, Texas, USA

${ }^{6}$ Pharmacy, University of Birmingham Edgbaston Campus, Birmingham, UK

Twitter Abdullah A Alhifany @aalhifany

Acknowledgements We would like to thank King Saud University, Riyadh Saudi Arabia for supporting this research project (RSP-2019/74).

Contributors AAA designed the research question. ARA, TAA, ANS, MA and FSA contributed to the searches, extraction of data and analysis. AAA, IA and EC contributed to the preparation of the manuscript.

Funding The authors have not declared a specific grant for this research from any funding agency in the public, commercial or not-for-profit sectors.

Competing interests None declared.

Patient consent for publication Not required.

Provenance and peer review Not commissioned; externally peer reviewed.

Data availability statement All data relevant to the study are included in the article or uploaded as online supplementary information.

Open access This is an open access article distributed in accordance with the Creative Commons Attribution Non Commercial (CC BY-NC 4.0) license, which permits others to distribute, remix, adapt, build upon this work non-commercially, and license their derivative works on different terms, provided the original work is properly cited, appropriate credit is given, any changes made indicated, and the use is non-commercial. See: http://creativecommons.org/licenses/by-nc/4.0/.

\section{ORCID iD}

Ejaz Cheema http://orcid.org/0000-0002-8538-7392

\section{REFERENCES}

1 Magill SS, Edwards JR, Bamberg W, et al. Multistate pointprevalence survey of health care-associated infections. N Engl J Med 2014;370:1198-208.

2 Zilberberg MD, Shorr AF, Kollef MH. Growth and geographic variation in hospitalizations with resistant infections, United States, 20002005. Emerg Infect Dis 2008;14:1756-8.

3 McFarland LV. Update on the changing epidemiology of Clostridium difficile-associated disease. Nat Clin Pract Gastroenterol Hepatol 2008;5:40-8.

4 Lessa FC, Mu Y, Bamberg WM, et al. Burden of Clostridium difficile infection in the United States. N Engl J Med 2015;372:825-34. 
5 Leffler DA, Lamont JT, infection Cdifficile. Clostridium difficile infection.. N Engl J Med 2015;372:1539-48.

6 Kuehne SA, Cartman ST, Heap JT, et al. The role of toxin A and toxin B in Clostridium difficile infection. Nature 2010;467:711-3.

7 Lyras D, O'Connor JR, Howarth PM, et al. Toxin B is essential for virulence of Clostridium difficile. Nature 2009;458:1176-9.

8 Smits LP, Bouter KEC, de Vos WM, et al. Therapeutic potential of fecal microbiota transplantation. Gastroenterology 2013;145:946-53.

9 Cammarota G, Masucci L, laniro G, et al. Randomised clinical trial: faecal microbiota transplantation by colonoscopy vs. vancomycin for the treatment of recurrent Clostridium difficile infection. Aliment Pharmacol Ther 2015;41:835-43.

10 Moayyedi P, Yuan Y, Baharith $\mathrm{H}$, et al. Faecal microbiota transplantation for $<$ em $>$ Clostridium difficile $</$ em $>$-associated diarrhoea: a systematic review of randomised controlled trials. Med $J$ Aust 2017;207:166-72.

11 Quraishi MN, Widlak M, Bhala N, et al. Systematic review with metaanalysis: the efficacy of faecal microbiota transplantation for the treatment of recurrent and refractory Clostridium difficile infection. Aliment Pharmacol Ther 2017:46:479-93.

12 van Nood E, Vrieze A, Nieuwdorp M, et al. Duodenal infusion of donor feces for recurrent Clostridium difficile. N Engl J Med 2013;368:407-15.

13 McDonald LC, Gerding DN, Johnson S, et al. Clinical practice guidelines for Clostridium difficile infection in adults and children: 2017 update by the infectious diseases Society of America (IDSA) and Society for healthcare epidemiology of America (SheA). Clin Infect Dis 2018;66:987-94.

14 Orenstein R, Dubberke E, Hardi R, et al. Safety and durability of RBX2660 (microbiota suspension) for recurrent Clostridium difficile infection: results of the punch CD study. Clin Infect Dis 2016;62:596-602.
15 Lowy I, Molrine DC, Leav BA, et al. Treatment with monoclonal antibodies against Clostridium difficile toxins. $N$ Engl $\mathrm{J}$ Med 2010;362:197-205.

16 Wilcox MH, Gerding DN, Poxton IR, et al. Bezlotoxumab for prevention of recurrent Clostridium difficile infection. N Engl J Med 2017;376:305-17.

17 Moher D, Liberati A, Tetzlaff J, et al. Preferred reporting items for systematic reviews and meta-analyses: the PRISMA statement. Int $J$ Surg 2010;8:336-41.

18 Higgins JPT, Green S. Cochrane Handbook for systematic reviews of interventions version. 5.1.0 (updated March 2011). The Cochrane collaboration. Available: http://handbook-5-1.cochrane.org/

19 Hota SS, Sales V, Tomlinson G, et al. Oral vancomycin followed by fecal transplantation versus tapering oral vancomycin treatment for recurrent Clostridium difficile infection: an open-label, randomized controlled trial. Clin Infect Dis 2017;64:265-71.

20 Leav BA, Blair B, Leney M, et al. Serum anti-toxin B antibody correlates with protection from recurrent Clostridium difficile infection (CDI). Vaccine 2010;28:965-9.

$21 \mathrm{Ng} \mathrm{S}$, Wong S, Lui R, et al. Vancomycin followed by fecal microbiota transplantation versus vancomycin for initial Clostridium difficile infection: an open-label randomised controlled trial. United European Gastroenterology Journal 2017:5

22 Camacho-Ortiz A, Gutiérrez-Delgado EM, Garcia-Mazcorro JF, et al. Randomized clinical trial to evaluate the effect of fecal microbiota transplant for initial Clostridium difficile infection in intestinal microbiome. PLoS One 2017;12:e0189768.

23 Waye A, Atkins K, Kao D. Cost averted with timely fecal microbiota transplantation in the management of recurrent Clostridium difficile infection in Alberta, Canada. J Clin Gastroenterol 2016;50:747-53.

24 Couture-Cossette A, Carignan A, llangumaran S, et al. Bezlotoxumab for the prevention of Clostridium difficile recurrence. Expert Opin Biol Ther 2017;17:1-7. 